\title{
Electron acceleration at comet 67P/Churyumov-Gerasimenko
}

\author{
R. Goldstein ${ }^{1}$, J. L. Burch ${ }^{1}$, K. Llera ${ }^{1}$, P. Mokashi ${ }^{1}$, H. Nilsson ${ }^{2}$, K. Dokgo ${ }^{1}$, A. Eriksson ${ }^{3}$, E. Odelstad ${ }^{3}$, and I. Richter ${ }^{4}$ \\ ${ }^{1}$ Southwest Research Institute, 6220 Culebra Road, San Antonio, TX 78238, USA \\ e-mail: rgoldstein@swri.edu \\ ${ }^{2}$ Swedish Institute of Space Physics, Kiruna, Sweden \\ ${ }^{3}$ Swedish Institute of Space Physics, Uppsala, Sweden \\ 4 Technical University of Braunschweig, Braunschweig, Germany
}

Received 21 November 2018 / Accepted 3 May 2019

\begin{abstract}
We report the observation by the Ion and Electron Sensor (IES) of energetic ( $>1 \mathrm{keV}$ ) electrons in the plasma environment of comet 67P Churyumov-Gerasimenko (67P). Most of the electrons in the cometary coma are expected to be of solar wind, photoionization, or electron impact origin and are therefore not expected to exceed some hundreds of eV in energy. During the Vega flybys of comet Halley, $1 \mathrm{keV}$ electrons were also observed, and these are explained as having been accelerated by lower hybrid (LH) waves resulting from the two-stream instability involving the solar wind and pickup-ion flows. These waves resonate with the cyclotron motion of the ions and the longitudinal motion of electrons and are on the order of several $\mathrm{Hz}$, at least in the case of 67P. We postulate that the energetic electrons we have observed intermittently during December 2015 through January 2016 are also the result of such a process and that Landau damping causes the acceleration and subsequent abrupt decrease in this energy (also seen at Halley). We show from this study an event on 19 January 2016 when IES simultaneously observed accelerated electrons, solar wind protons, water ions, and LH waves. A dispersion analysis shows that the ion-ion two-stream instability has positive growth rates for such waves during the observation period.
\end{abstract}

Key words. plasmas - waves - methods: data analysis - comets: individual: 67P/Churyumov-Gerasimenko

\section{Introduction}

The Ion and Electron Sensor (IES; Burch et al. 2007) as well as other instruments of the Rosetta Plasma Consortium (RPC; Carr et al. 2007) on board the Rosetta spacecraft (S/C) measured the characteristics of the solar wind and locally produced plasma almost continuously from arrival at comet 67P/ChuryumovGerasimenko (67P) in August 2014 until the end of the mission on 30 September 2016. The locally produced plasma is largely caused by solar ultraviolet photo-ionization of the neutral gas emitted by the comet nucleus, as a result of solar heating as the comet moved closer to the Sun (see, e.g., Nilsson et al. 2015a,b and Goldstein et al. 2015). The energy of these photoelectrons is generally some tens of eV (see also e.g., Galand et al. 2016 for details of processes in the coma). This study reports on measurements by IES of $1 \mathrm{keV}$ electrons on 19 January 2016 when Rosetta was past perihelion and moving outward from the Sun at a distance of about 2 AU. During this period, the S/C was in terminator orbits $80-100 \mathrm{~km}$ from 67P. Figure 1 shows an example of an electron energy-time spectrogram for 19 January 2016, measured by IES. Most of the electrons measured are lower than $200 \mathrm{eV}$, but there are intermittent periods of electrons that have energies up to $>1 \mathrm{keV}$ (the white signal that appears intermittently at about $150 \mathrm{eV}$ probably is the result of interference from some external source on Rosetta).

The history of measurements of energetic electrons at comets includes a report by Hall et al. (1986), who described

\footnotetext{
* The movie associated to Fig. 6 is available at https://www. aanda.org
}

the electrons that were measured up to $1 \mathrm{keV}$ after the September 1984 Active Magnetospheric Particle Tracer Explorers (AMPTE) mission (Haerendel 1985) releases of Li in the solar wind. As is the case for photoelectrons at 67P, the electrons produced from ionization of Li would also be of low energy, which raised the question about the mechanism that energized the electrons. One suggestion discussed in the Hall et al. (1986) report was wave-particle interactions, specifically by the electrostatic lower hybrid (LH) waves. These waves can be generated by the ion-ion two-stream instability involving solar wind and pickupion flows. In the presence of a magnetic field, LH waves resonate with the cyclotron motion of the ions and the longitudinal motion of electrons. The existence of such waves in the AMPTE release environment was reported by Häusler et al. (1986). During the VEGA2 flyby of comet Halley, Gringauz et al. (1986) measured electrons up to $1 \mathrm{keV}$. One characteristic of these electrons was a relatively flat plateau in energy followed by a sharp drop in count rate at the highest energy. Also from VEGA2, Klimov et al. (1986) had measured electrostatic waves in the LH frequency range. Subsequently, Shapiro et al. (1999) explained the acceleration of the electrons measured at Halley in terms of LH waves. When the velocity of the electrons coincides with the parallel phase velocity of the wave, the electrons stay for a longer time in phase resonance with the wave, thereby gaining much energy from it. This is the so-called Cherenkov wave-particle resonance condition, given by Eq. (5) of Shapiro et al. (1999). For the conditions at 67P, this process results in a resonant energy of at least a few hundred eV with an LH frequency of about $13 \mathrm{~Hz}$. Karlsson et al. (2017) and André et al. (2017) have studied waves at $67 \mathrm{P}$ and identified cases of observing these waves 


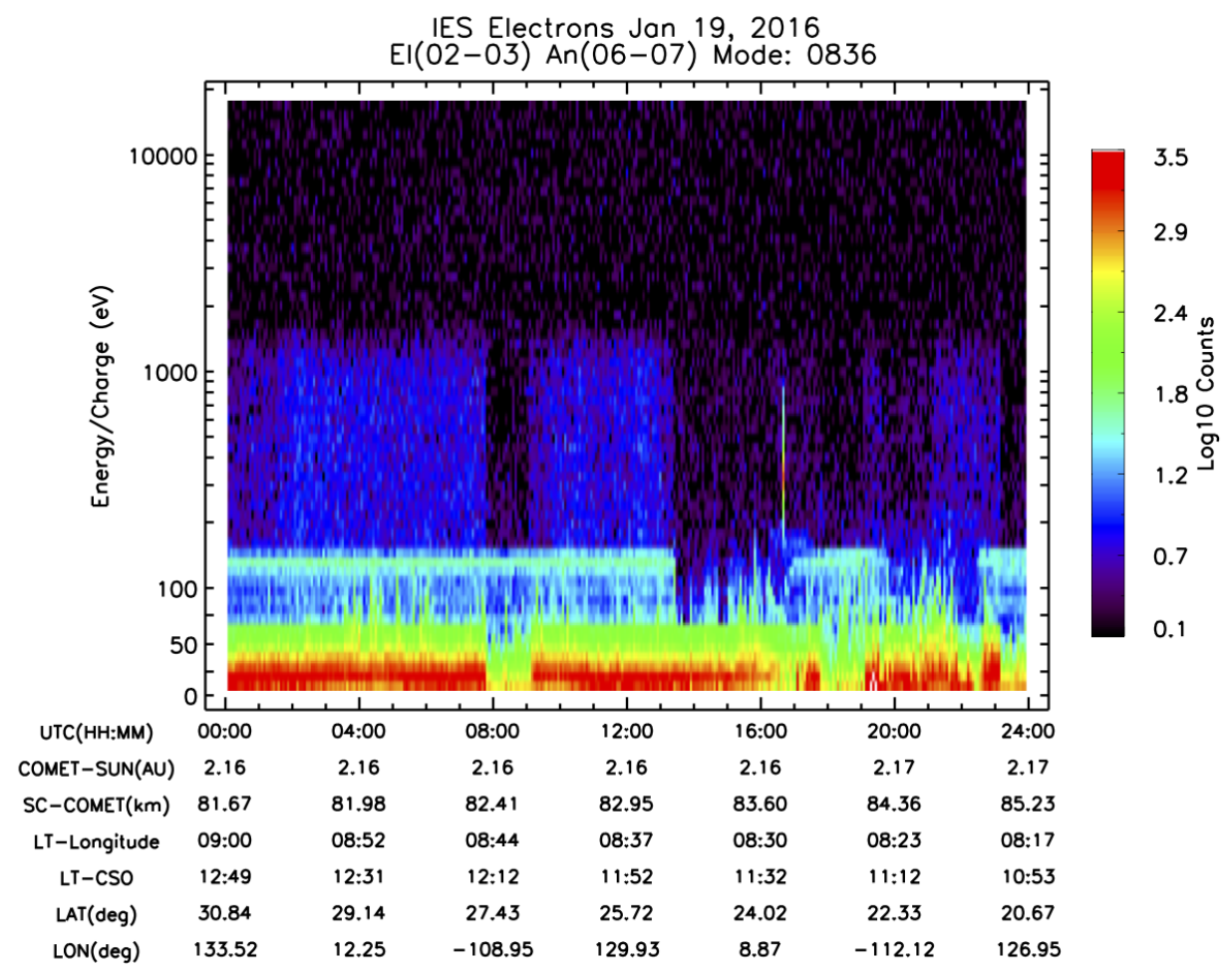

Fig. 1. Energy-time spectrogram for IES electrons on 19 Jan 2016. Note the intermittent periods of electrons with energy up to about $1 \mathrm{keV}$.

January 19, 2016: Ion Counts

Counts have been summed over Elevations and Azimuth

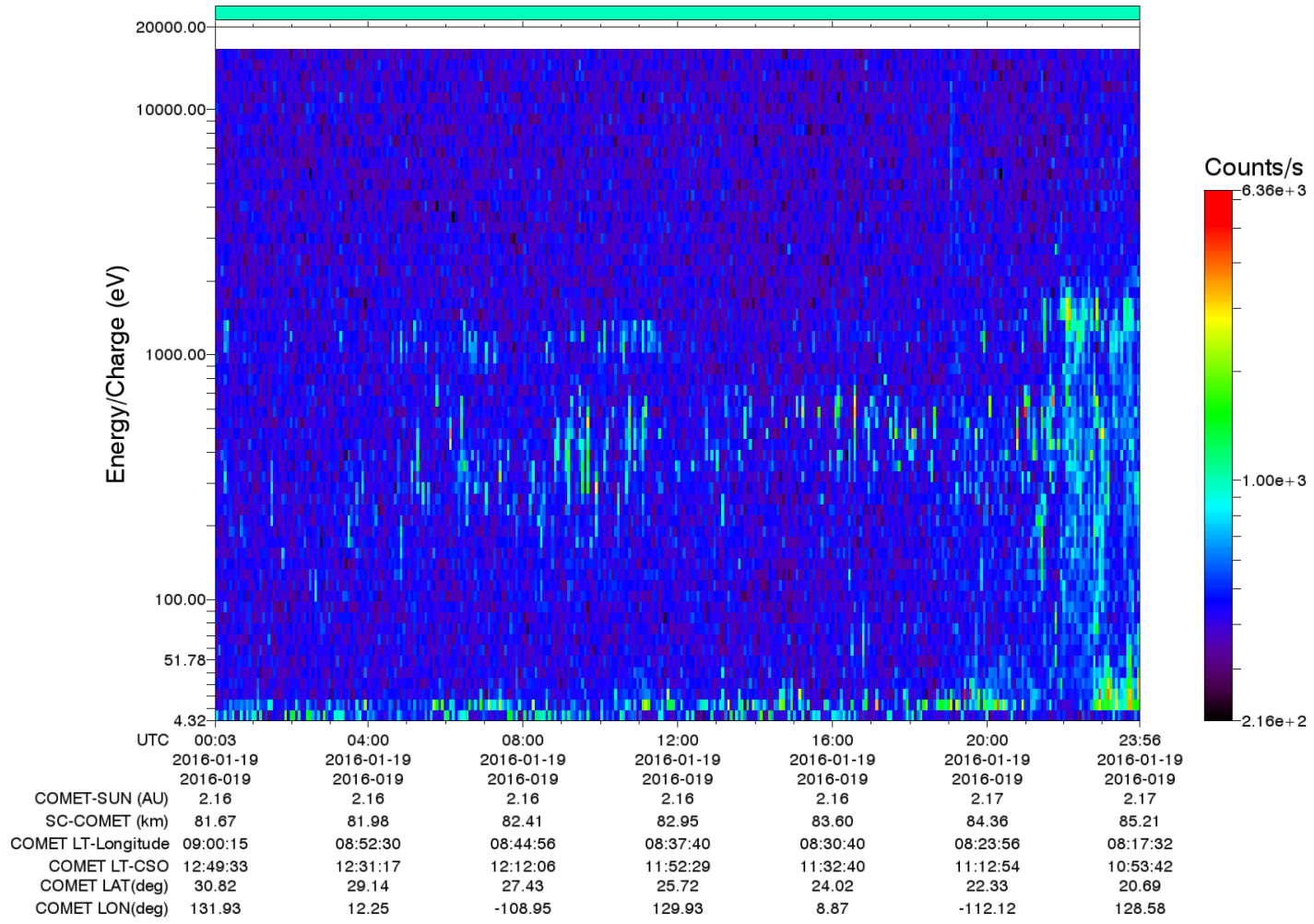

Fig. 2. Energy-time spectrogram for IES on 19 Jan 2016.

measured by LAP, the Langmuir Probe (Eriksson et al. 2007) and MIP, the Mutual Impedance Probe (Trotignon et al. 2007), both investigations members of the Rosetta Plasma Consortium (RPC).
In the following sections we describe the analysis of the ion and electron data and the results of wave growth rate calculations. They both show positive values for the observed solar wind fluxes. 


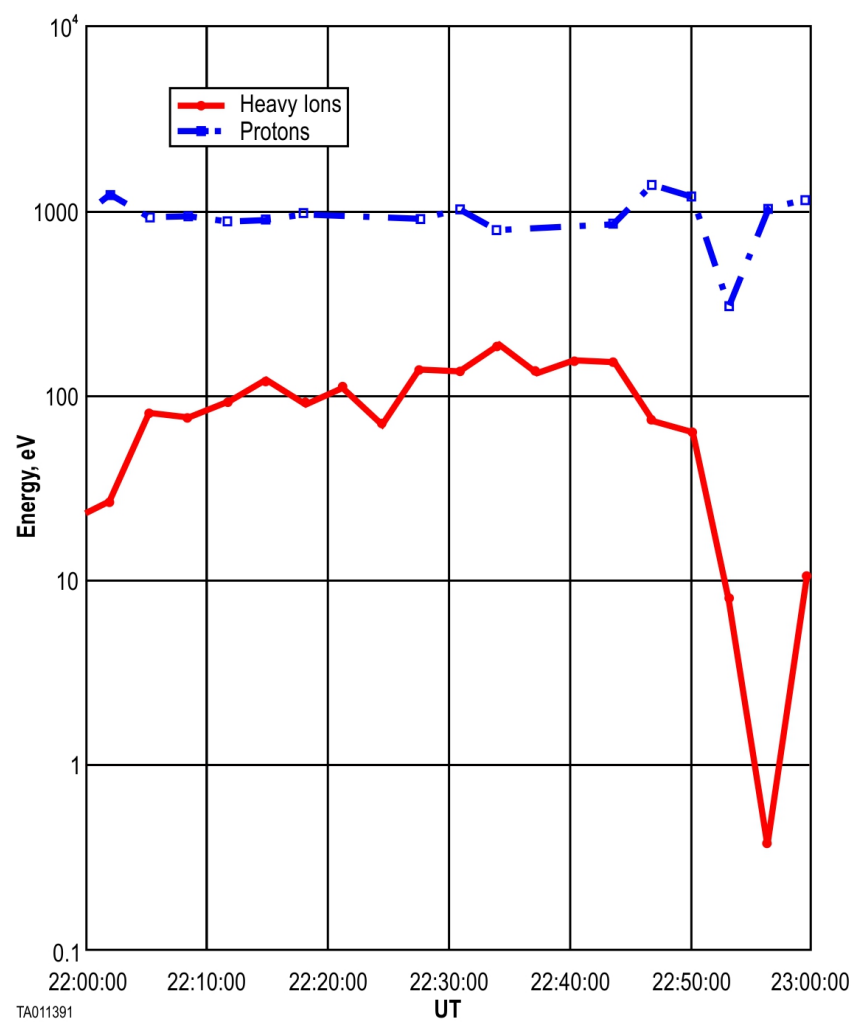

Fig. 3. Bulk energy of protons (blue) and heavy ions (red, assumed $\mathrm{H}_{2} \mathrm{O}^{+}$).

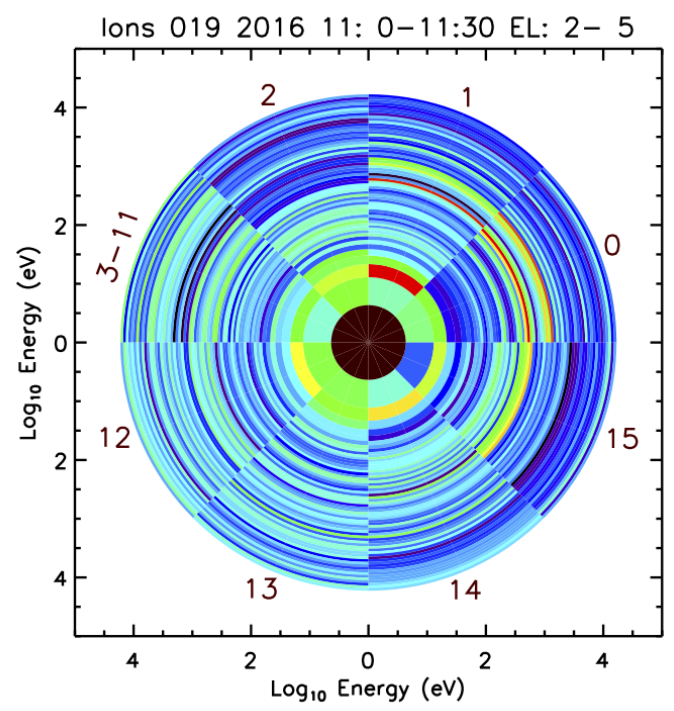

Fig. 4. IES polar ion plot for 19 January 2016, 11:00-11:30 UT. Sun is in $(3-11)$, and $67 \mathrm{P}$ is at 0 .

\section{Measurements}

\subsection{Ions}

Because ions play a role in transferring energy to the electrons, identifying the ion mass, energy, and flow direction is necessary to understand details of the process. IES measured ions over an energy/charge range from $4 \mathrm{eV} \mathrm{e}^{-1}$ to $17.67 \mathrm{keV} \mathrm{e}^{-1}$ but did not measure ion mass (Burch et al. 2007). Under conditions when the solar wind and little else in the way of ions was present in the IES field of view (FOV), it was straightforward to identify protons and alphas, and often $\mathrm{He}^{+}$as well. Under other conditions,

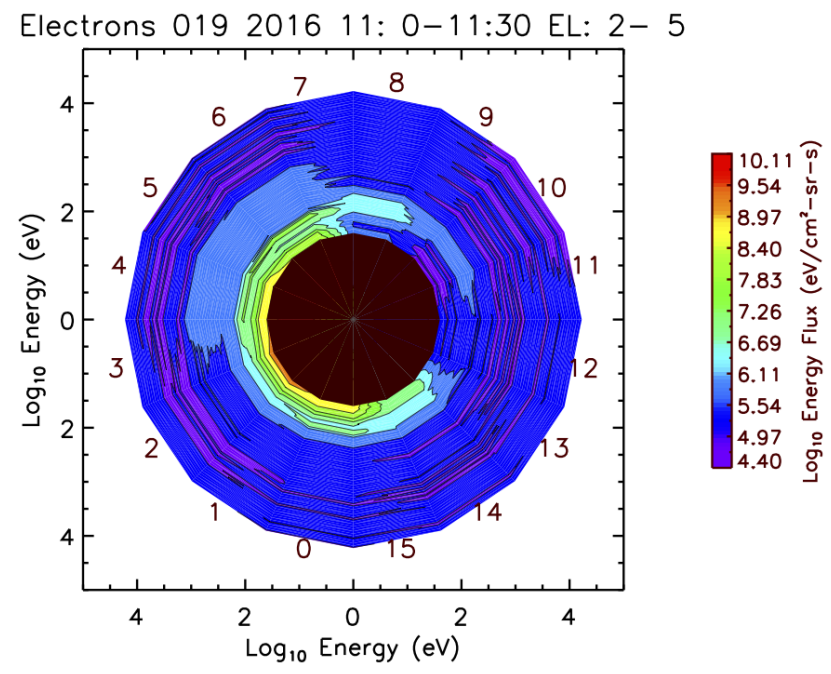

Fig. 5. IES polar electron plot for 19 January 2016, 11:00-11:30 UT. Sun is between anodes 8 and 9 , and 67P is between 0 and 15 .

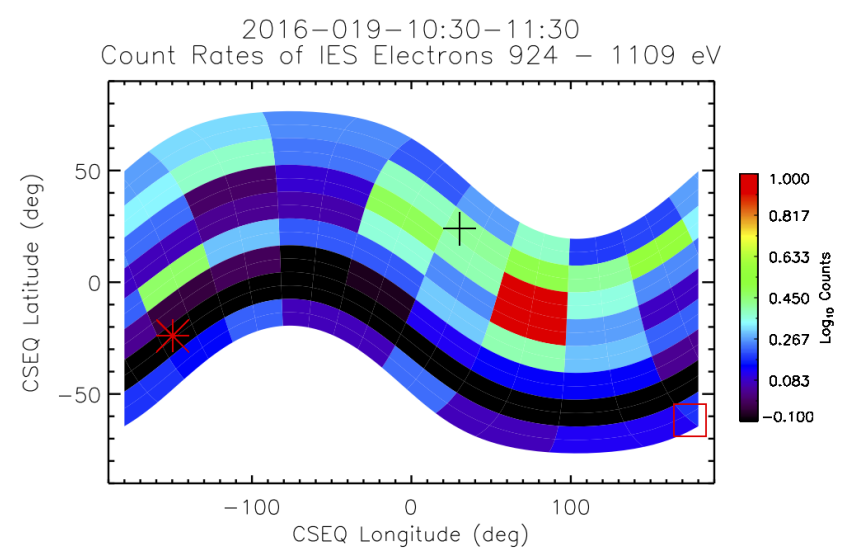

Fig. 6. CSEQ plot of $16 \times 16$ IES electron pixel count-rates for the energy range 924-1109 eV for 19 January 2016, 10:30-11:30 UT. The red square gives the location of 67P in the IES FOV. The black plus and the red asterisk give the location of the intersection of the average magnetic field vector and the IES FOV. Movie available online.

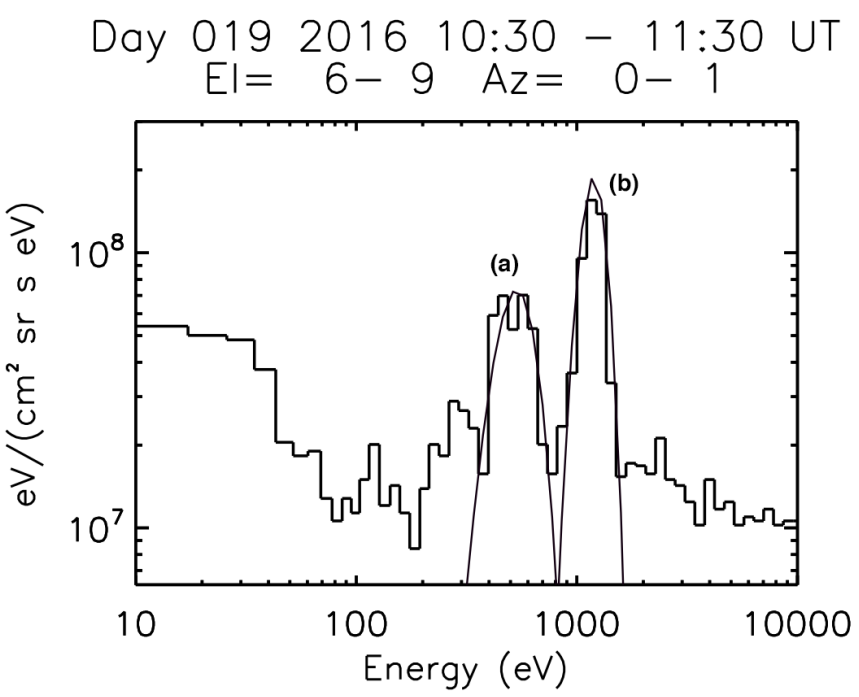

Fig. 7. Drifting Maxwellian fits to the ion flux from the data of Fig. 2 for the period 10:30-11:30 UT and the IES look directions indicated. Water ions are in the peak labeled (a) and solar wind ions are in the peak labeled (b). For water, the flow velocity was $72 \mathrm{~km} \mathrm{~s}^{-1}$, the density was $4.0 \mathrm{~cm} \mathrm{~s}^{-3}$, and the temperature was $12 \mathrm{eV}$. For protons, the velocity was $470 \mathrm{~km} \mathrm{~s}^{-1}$, and the density was $0.35 \mathrm{~cm} \mathrm{~s}^{-3}$. 

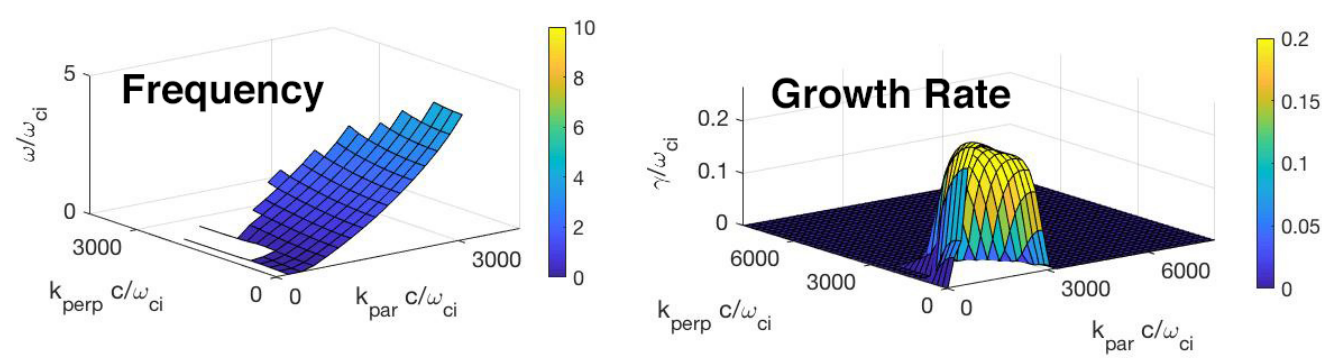

Fig. 8. Results of wave growth calculations for the interaction of the water and proton flows of Fig. 7.

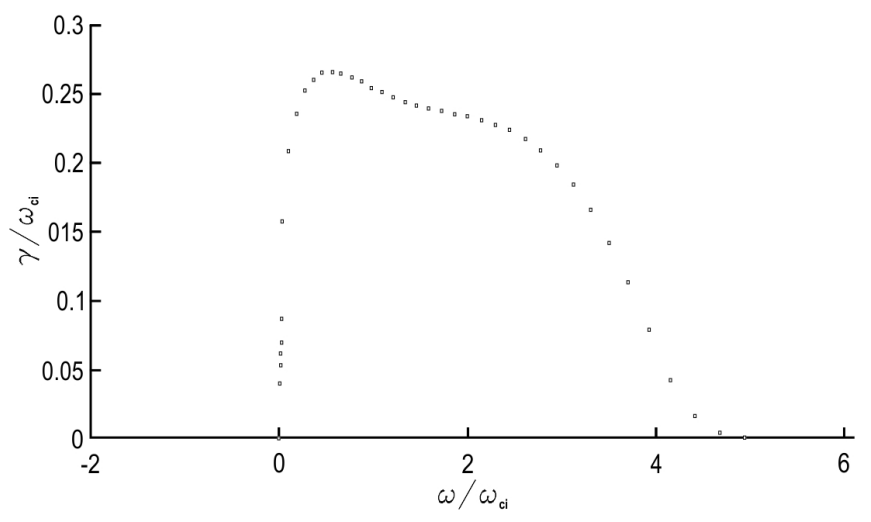

Fig. 9. Data from Fig. 8 plotted as growth rate vs. frequency.

it is necessary to rely on mass estimates or other measurements (Nilsson et al. 2007). Figure 2 is an IES ion energy-time spectrogram for 19 January 2016. The lowest energy ions that appear here are probably those produced very near Rosetta by photoionization of the neutral gas and attraction to the $\mathrm{S} / \mathrm{C}$ by its negative potential. This potential is often negative, as measured by LAP (Eriksson et al. 2007). Until 20:00 UT, ions of energy $\approx 500 \mathrm{eV}$ and $1 \mathrm{keV}$ are present. The ion spectrogram shown in Fig. 2 probably indicates that both the solar wind protons and lower energy water ions show an unstable flow, which is one of the requirements for the electron acceleration by the process that we propose.

Based on a comparison with ICA when that instrument obtained suitable data, we suggest that the latter are solar wind protons, while the lower energy ions with a broader distribution are heavier pickup-ions. At 21:00 until the end of this day, the $1 \mathrm{keV}$ ions appear more intense, and a broader energy range of ions appears at lower energy. These lower energy ions are likely ions that have been picked up by the solar wind at some distance from Rosetta and accelerated from their original low energy at their creation as they are brought back toward the S/C by the solar wind. ICA was able to distinguish some of the higher masses during the later part of this day, and Fig. 3 shows resulting energy-time plots of solar wind protons and what is assumed to be water ions (measured by ICA). The proton energy in this figure is in agreement with the IES measurement shown in Fig. 2. Figure 4 is an example of an ion polar spectrogram plot for 11:00-11:30 UT on 19 January 2016 from IES data in IES coordinates. Energy is along the radius, and azimuth (anode number) is along the circumference. During that time period, the Sun was toward anodes 2 and 3-11, and 67P was between anodes 13 and 14. Low-energy ions are seen from the solar direction, but $1 \mathrm{keV}$ ions were $90-135^{\circ}$ from the solar direction. We identify these ions as solar wind protons. The broad peak at a few hundred $\mathrm{eV}$ is probably caused by pickup-ions, probably mostly $\mathrm{H}_{2} \mathrm{O}^{+}$, based on the energy range of protons and water ions as seen by ICA between 22 and 23 UT.

\subsection{Electrons}

An electron polar spectrogram plot similar to that for ions is shown in Fig. 5. The Sun is at anode 9, and 67P at anodes 0 and 15. (The locations of the electron anodes relative to the Sun and 67P are different from those of the ions.) The low-energy electrons, presumably resulting from photoionization of nucleus outgassing products, are spread over much of the instrument FOV. The $\approx 1 \mathrm{keV}$ electrons, the primary subject of this study, appear at anodes 4-7, roughly moving anti-sunward. Figure 6 shows the electron count rate in the energy range 924-1069 eV in the CSEQ system, showing the IES $\pm 45^{\circ}$ elevation by $360^{\circ}$ azimuth FOV. This is the cometocentric solar equatorial system. The Sun is at coordinates $(0,0)$, and the red square designates the location of 67P in the IES FOV. The black plus and red asterisk show the intersection of the magnetic field vector (averaged over 10:30 to 11:30 UT) measured by the onboard magnetometer system, RPC-MAG (Glassmeier et al. 2007). The black band in the lower part of the figure is the location of an azimuth bin for which data have been deleted because of the very low signalto-noise ratio $(\mathrm{S} / \mathrm{N})$ in that bin. The movie associated to Fig. 6 is available online. The high count-rate in this video is beamlike, maintaining a direction with latitude near $0^{\circ}$, and longitude $+80^{\circ}$ beginning at energy $500 \mathrm{eV}$ and continuing to $1109 \mathrm{eV}$. Figure 6 shows one frame of this beam. At higher energies, the high count-rate is no longer so focused on a single pixel. We suggest that this beam-like behavior is the result of the excitation of the electrons by wave activity, to be discussed in the next section.

\section{Electron-excitation process}

Many wave processes can occur in a plasma. For example, the lower hybrid drift instability (LHDI, Gladd 1976) is caused by plasma pressure gradients near the comet. While Landau damping can occur, the resulting electron energies would be very low (probably lower than $10 \mathrm{eV}$ ). For keV electrons, Landau damping would have to occur over larger distances, estimated at $10^{4} \mathrm{~km}$ by Shapiro et al. (1999). At these distances from the comet, the LHDI would not be active, but the two-stream instability between solar wind ions and pickup-ions would be. The frequency ranges of lower hybrid waves would be the same, but the dispersion relation for the two-stream instability is different from that of the LHDI, so that the propagation characteristics should be different (Gladd 1976). In particular, the two-stream instability, with which we are concerned here, produces strongly obliquely propagating waves.

Figure 7 shows drifting Maxwellian fits to the water ion (a) and proton (b) fluxes of the data of Fig. 3 for the period 10:30 to 11:30 UT and the IES look directions indicated. Species selection is based on energy range, so that not all signal shown is relevant. Therefore, the indicated fits are quite good, although much signal lies also outside the region fitted with a drifting Maxwellian distribution. This is in particular true for the solar 

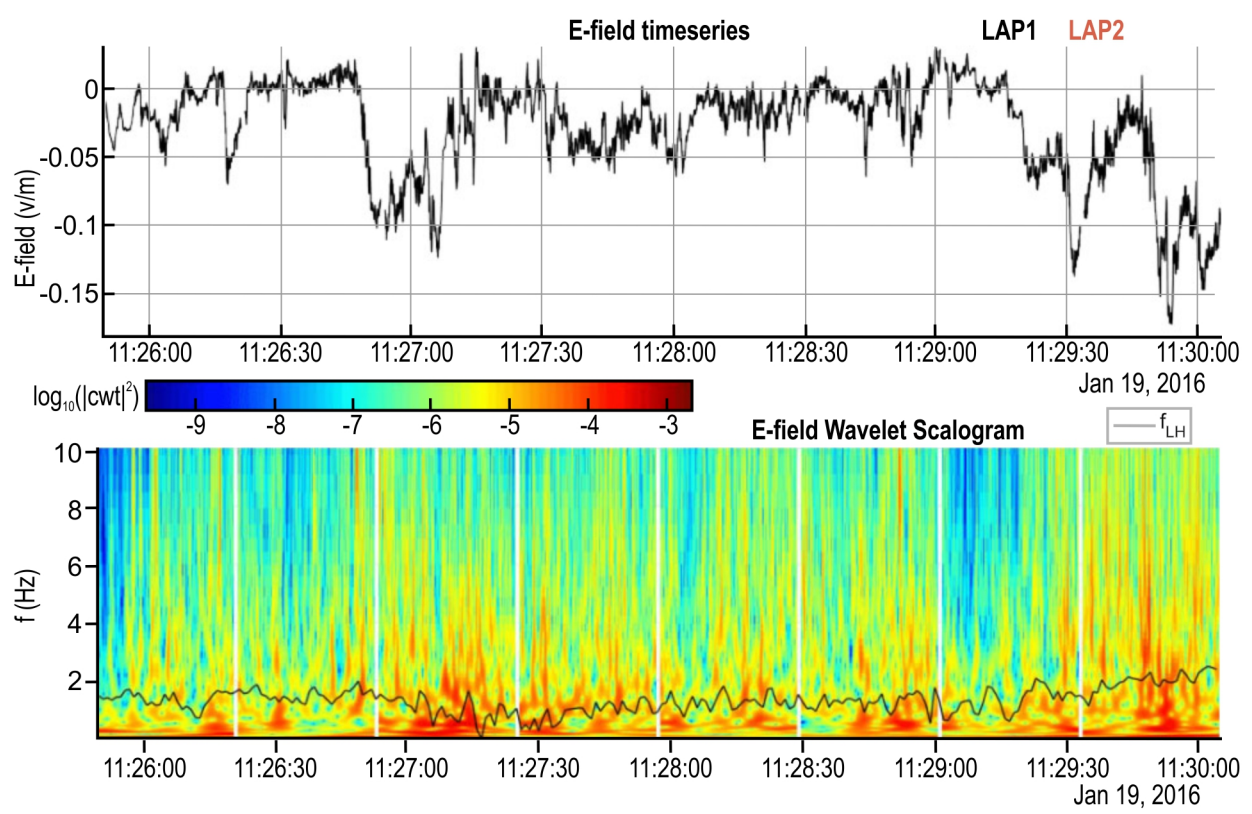

Fig. 10. Electric waves measured by the Langmuir probe on 19 January 2016 at the end of the interval 10:30 to $11: 30$. Top plot: measured electric field $\left(\mathrm{V} \mathrm{m}^{-1}\right)$. Bottom plot: scalogram of the resulting frequency. The black line near the bottom gives the LH wave frequency.

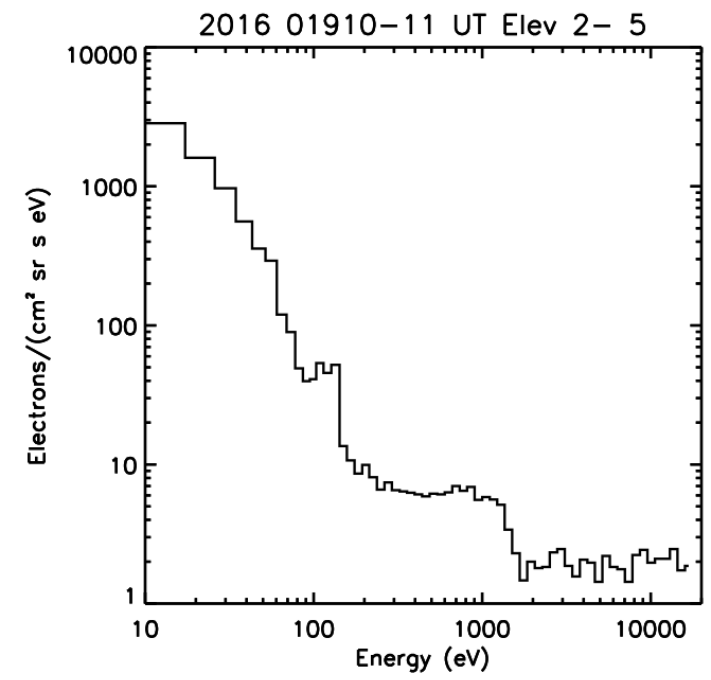

Fig. 11. Energy dependence of electron flux summed over 10:3011:30 UT 19 January 2016.

wind. For the cometary ions the fit is less certain, but still indicative of the effective temperature of the water ion population. Based on these fits, the flow velocity for water was $72 \mathrm{~km} \mathrm{~s}^{-1}$, the density was $4.0 \mathrm{~cm}^{-3}$, and the temperature was $12 \mathrm{eV}$. For protons, velocity was $470 \mathrm{~km} \mathrm{~s}^{-1}$, density $0.35 \mathrm{~cm}^{-3}$, and temperature was $12 \mathrm{eV}$. The interaction between these two flows can result in a two-stream instability that generates the LH waves that transfer energy from the ions to the electrons. We have used the kinetic dispersion relation solver PDRK (Xie \& Xiao 2016) to estimate the wave growth rate resulting from the interaction between the solar wind protons and the picked-up $\mathrm{H}_{2} \mathrm{O}^{+}$ions from 67P. The calculated rates are shown in Fig. 8. The left plot gives the frequency, and the right plot the growth rate. All parameters are normalized by the proton cyclotron frequency $\omega_{\text {ci }}$. This frequency is $\approx 0.1 \mathrm{~Hz}$ for a $20 \mathrm{nT}$ field. Data from Fig. 8 are plotted as growth rate versus frequency in Fig. 9, showing the maximum rate at about $3 \mathrm{~Hz}$. Using data from LAP (Eriksson et al. 2007) for 18 November 2015, André et al. (2017) found waves of frequency $\approx 5 \mathrm{~Hz}$, which is in the range of LH waves and on the same order as the $3 \mathrm{~Hz}$ calculated above for the measurements reported here. We did not observe energetic electrons during that day. However, LH waves were seen during the period of the accelerated electrons observed on 19 January 2016, as shown in Fig. 10 for a selected period of that interval. This shows the measured electric field $\left(\mathrm{V} \mathrm{m}^{-1}\right)$ at the top and the resulting wave frequency $(\mathrm{Hz})$ at the bottom. The black line shows the LH wave frequency. The $\approx 1 \mathrm{keV}$ electrons we have measured were probably accelerated by LH waves resulting from the two-stream instability between the solar wind protons and picked-up water ions. See Fig. 3 for ICA measurements of these two ions at that time. Figure 11 shows a plot of the energy dependence of electron flux for 22:00 to 23:00 on 19 January 2016. A plateau occurs from about $300 \mathrm{eV}$ to a sharp dropoff at about $1500 \mathrm{eV}$. This plateau is similar to the results of electron measurements by Gringauz et al. (1986) at comet Halley; see in particular Fig. 6 in that reference. Shapiro et al. (1999) later showed that the plateau is closely reproduced with results of an analysis of assumed LH waves that resulted from interaction of the flows of solar wind protons and pickup water ions.

\section{Summary and conclusions}

The IES instrument has measured electrons of energy up to $\approx 1 \mathrm{keV}$ with a sharp dropoff above that. IES simultaneously observed these accelerated electrons, unstable flow of solar wind protons and water ions, and LH waves, over the period 10:30-11:30 UT. A dispersion analysis showed that the ionion two-stream instability has positive growth rates for such waves. This observation is similar to those made at comet Halley by Gringauz et al. (1987). The explanation by Shapiro et al. (1999) was that the energy came from the solar wind as a result of the two-stream instability with pickup-ions, producing lower hybrid frequency waves. These waves then accelerate the electrons, but the electrons cannot exceed the wave phase velocity. This results in the sharp dropoff in energy, analogous to a surfer falling off a wave.

With Rosetta IES data we have identified the source of LH waves as the ion-ion two-stream instability between solar wind protons and picked-up water ions. Simultaneous measurements of LH waves on board Rosetta confirms the result. The plateautype electron energy spectrum is similar to that observed at 
Halley (Gringauz et al. 1986, 1987) and in the laboratory as a result of the lower hybrid current drive, which is used to heat electrons in magnetic-confinement fusion (Fisch 1987).

It is notable that although André et al. (2017) found waves of LF frequency, as mentioned above, we did not observe accelerated electrons at that time. The question remains, then, whether other factors are necessary for the acceleration process to take place.

Acknowledgements. Rosetta is an ESA mission with contributions from its member states and the U.S. National Aeronautics and Space Administration (NASA)

The work on IES was supported by NASA through contract 1345493 with the Jet Propulsion Laboratory, California Institute of Technology. Work on the ICA and LAP instruments was supported by the Swedish National Space Board. Work on the MAG instrument was financially supported by the German Ministerium fü Wirtschaft und Energie and the Deutsche Zentrum für Luft- und Raumfart under contract 50 QP 1401. We thank the teams at Imperial College London and ESA, who have been responsible for the operation of our instruments. The authors thank the referee for several helpful suggestions.

\section{References}

André, M., Odelstad, E., Graham, D. P., et al. 2017, MNRAS, 469, S29 Burch, J. L., Goldstein, R., Cravens, T. E., et al. 2007, Space Sci. Rev., 128, 697 Carr, C., Cupido, E., Lee, C. G. Y., et al. 2007, Space Sci. Rev., 128, 629
Eriksson, A. I., Boström, R., Gill, R., et al. 2007, Space Sci. Rev., 128, 729

Fisch, N. J. 1987, Rev. Mod. Phys., 59, 175

Galand, M., Héritier, K. L., Odelstad, E., et al. 2016, MNRAS, 462, S331

Gladd, N. T. 1976, Plasma Phys., 18, 27

Glassmeier, K.-H., Richter, I., Diedrich, A., et al. 2007, Space Sci. Rev., 128, 649

Goldstein, R., Burch, J. L., Mokashi, P., et al. 2015, Geophys. Res. Lett., 42, 3093

Gringauz, K. I., Gombosi, T. I., Remizov, A. P., et al. 1986, Nature, 321, 282

Gringauz, K. I., Remizov, A. P., Verigin, M. I., et al. 1987, A\&A, 187, 287

Haerendel, G. 1985, Johns Hopkins APL Tech. Dig., 6, 263

Hall, D. S., Bryant, D. A., Chaloner, C. P., Bingham, R., \& Lepine, D. R. 1986, J. Geophys. Res., 91, 1320

Häusler, B., Woolliscroft, L. J., Anderson, R. R., et al. 1986, J. Geophys. Res., 91, 1283

Karlsson, T., Eriksson, A. I., Odelstad, E., et al. 2017, Geophys. Res. Lett., 44, 1641

Klimov, S., Savin, S., Aleksevich, Y., et al. 1986, Nature, 321, 292

Nilsson, H., Lundin, R., Lundin, K., et al. 2007, Space Sci. Rev., 128, 671

Nilsson, H., Stenberg Wieser, G., Behar, E., et al. 2015a, Science, 347, aaa0571

Nilsson, H., Stenberg Wieser, G., Behar, E., et al. 2015b, A\&A, 583, A20

Shapiro, V. D., Bingham, R., Dawson, J. M., et al. 1999, J. Geophys. Res., 104, 2537

Trotignon, J. G., Michau, J. L., Lagoutte, D., et al. 2007, Space Sci. Rev., 128, 713

Xie, H., \& Xiao, Y. 2016, Plasma Sci. Technol., 18, 97 